INPLASY

PROTOCOL

To cite: Guo et al. Thunder-Fire Moxibustion for Epigastralgia: A Protocol of Systematic Review and Meta-analysis of Randomized Controlled Trials. Inplasy protocol 202080101. doi:

10.37766/inplasy2020.8.0101

Received: 24 August 2020

Published: 24 August 2020

Corresponding author: Jun Xiong

xiongjun196071@163.com

Author Affiliation:

Jiangxi University of

Traditional Chinese Medicine

Support: 1050Project:

5141900101.

Review Stage at time of this submission: The review has not yet started.

Conflicts of interest:

The authors declare no conflicts of interest.

\section{Thunder-Fire Moxibustion for Epigastralgia: A Protocol of Systematic Review and Meta-analysis of Randomized Controlled Trials}

Guo, $\mathrm{H}^{1}$; Xiong, J2; Zhou, XC³.

Review question / Objective: To evaluate the effectiveness and safety of thunder-fire moxibustion for epigastralgia patients.

Condition being studied: The strategical calls the stomachache, what has the characteristics of a high incidence and recurrent, is often has the ache primarily illness by the stomach cavity near pit of the stomach place. Epigastralgia is common in acute and chronic gastritis, gastric and duodenal ulcer disease, gastric neurosis, etc. The major medications treating epigastralgia cause side effects, while there is evidence show that thunder-fire moxibustion (TFM) is a safe and effective treatment for epigastralgia.

INPLASY registration number: This protocol was registered with the International Platform of Registered Systematic Review and Meta-Analysis Protocols (INPLASY) on 24 August 2020 and was last updated on 24 August 2020 (registration number INPLASY202080101).

\section{INTRODUCTION}

Review question / Objective: To evaluate the effectiveness and safety of thunder-fire moxibustion for epigastralgia patients.

Condition being studied: The strategical calls the stomachache, what has the characteristics of a high incidence and recurrent, is often has the ache primarily illness by the stomach cavity near pit of the stomach place. Epigastralgia is common in acute and chronic gastritis, gastric and duodenal ulcer disease, gastric neurosis, etc. The major medications treating epigastralgia cause side effects, while 
there is evidence show that thunder-fire moxibustion (TFM) is a safe and effective treatment for epigastralgia.

\section{METHODS}

Participant or population: Patients with epigastralgia.

Intervention: Thunder-fire moxibustion, thunder-fire moxibustion combined with traditional Chinese medicine or other conventional treatment.

Comparator: Traditional Chinese medicine or other conventional treatment.

Study designs to be included: Randomized controlled trials (RCTs) which assessed the efficacy and safety of thunder-fire moxibustion for epigastralgia will be included.

Eligibility criteria: Subjects: patients with epigastralgia, age and sex were not restricted. Intervention measures: thunderfire moxibustion or thunder-fire moxibustion combined with traditional Chinese medicine or thunder-fire moxibustion combined with other conventional treatment were used as the intervention measures in the treatment group, while traditional Chinese medicine or other conventional treatment was used as the intervention measures in the control group Outcome measures: effective rate, recurrence rate, adverse reactions, cure rate, improvement of clinical symptoms.

Information sources: Pubmed, Embase, Cochrane Library, Chinese Biomedical Literatures Database(CBM), China National Knowledge Infrastructure (CNKI), WangFang Database (WF), Chinese Scientifific Journal Database (VIP).

Main outcome(s): Effective rate, recurrence rate, adverse reactions.

Additional outcome(s): Cure rate, improvement of clinical symptoms.

Quality assessment / Risk of bias analysis: According to the improved Jadad scoring scale, the quality of the included literature was evaluated. 1-3 were classified as low quality and 4-7 as high quality. Risk of bias(quality) assessment included randomised studies will be assessed for risk of bias by two independent raters(GH/ ZXC) using the Cochrane Collaboration's tool for assessing risk of bias in randomised trials. Any disagreements will be resolved through discussion or consultation with a third reviewer(XJ).

Strategy of data synthesis: RevMan $\mathbf{5 . 4}$ software (Cochrane Collaboration) was used for the meta-analysis. Dichotomous data were reported as risk ratio (RR) with 95\% confidence intervals (CI), while continuous data were reported as standardized mean difference (SMD) with $95 \%$ Cls. The Higgins $\left.\right|^{2}$ test was used to test heterogeneity with a significance level set at $\mathbf{5 0 \%}$. If heterogeneity was not significant $\left(I^{2} \leq 50 \%\right)$, the fixed effects model was used for meta-analysis. Otherwise, the random effects model was used $\left(\left.\right|^{2} \geq 50 \%\right)$. If possible, we investigated the potential explanations for heterogeneity and conducted subgroup analysis.

Subgroup analysis: If the necessary data are available, subgroup analysis will be carried out according to different factors as follows: 1. Control interventions (eg, traditional Chinese medicine, other conventional treatment) 2 . Interventions of experimental group (eg, thunder-fire moxibustion, thunder-fire moxibustion combined with traditional Chinese medicine, thunder-fire moxibustion combined with other conventional treatment) 3. Outcome indicators (eg, effective rate, recurrence rate, adverse reactions).

Sensibility analysis: Sensitivity analysis will be conducted in primary outcomes to test the result homogeneity. We will perform meta-analysis again after eliminate studies in low quality and use different statistical methods.

Country(ies) involved: China. 
Keywords: Epigastralgia; Thunder-Fire Moxibustion; Randomized Controlled Trials.

Contributions of each author:

Author 1 - Han Guo - The author drafted the manuscript and did statistical work.

Author 2 - Jun Xiong - Gave the conception and reviewed the manuscript, provided feedback for the manuscript and do the supervision work.

Author 3 - XingChen Zhou - Contributed to the development of the selection criteria, and the risk of bias assessment strategy. 